\title{
Actively reconfigurable compact vortex beam emitters
}

\author{
Michael J. Strain ${ }^{1,2}$, Huanlu $\mathrm{Li}^{5}$, Xinlun Cai ${ }^{3,4}$, Laura Meriggi ${ }^{2}$, Jianwei Wang ${ }^{5}$, Jiangbo Zhu ${ }^{4,5}$, \\ Kenan Cicek $^{5}$, Lifeng Chen ${ }^{5}$, Martin L. Garcia ${ }^{5}$, Mark G Thompson ${ }^{3}$, Marc Sorel ${ }^{2}$, Siyuan Yu ${ }^{4,5}$ \\ 1. Institute of Photonics, University of Strathclyde, Glasgow, G4 0NW, U.K. \\ 2. School of Engineering, University of Glasgow, Glasgow, G12 8LT, U. K. \\ 3. Centre for Quantum Photonics, University of Bristol, Bristol, UK. \\ 4. State Key Laboratory of Optoelectronic Mat. and Technologies and School of Physics and Engineering, Sun Yatsen University, \\ Guangzhou, China. \\ 5. Photonics Group, Merchant Venturers School of Engineering, University of Bristol, UK
}

\begin{abstract}
Electrical switching of the orbital angular momentum (OAM) mode order emitted from an SOI microring resonator is reported. The integration of the microring with waveguide couplers is used to demonstrate modulation of the relative phase between pairs of OAM modes with opposite signs.
\end{abstract}

The generation and manipulation of optical vortex beams has recently been the focus of intensive research efforts. The application of beams carrying orbital angular momentum (OAM) has been demonstrated in OAM mode order multiplexing for optical communications [1] and in optical tweezing and particle manipulation [2]. Furthermore, the generation of vortex beams has been shown in integrated devices fabricated on a silicon photonic platform [3,4]. This integrated generation approach not only allows compact device footprint and spatial multiplexing but has also demonstrated OAM mode order selectivity with injection wavelength as a parameter. Although this degree of freedom is useful, switching and reconfigurability of the OAM order at a single wavelength is highly desirable for integrated OAM multiplexing within WDM communications systems. In this work, electrical control is demonstrated on the OAM mode order of a single wavelength signal from a compact vortex beam emitter on silicon using a simple resistive heating element, as shown in Fig.1.

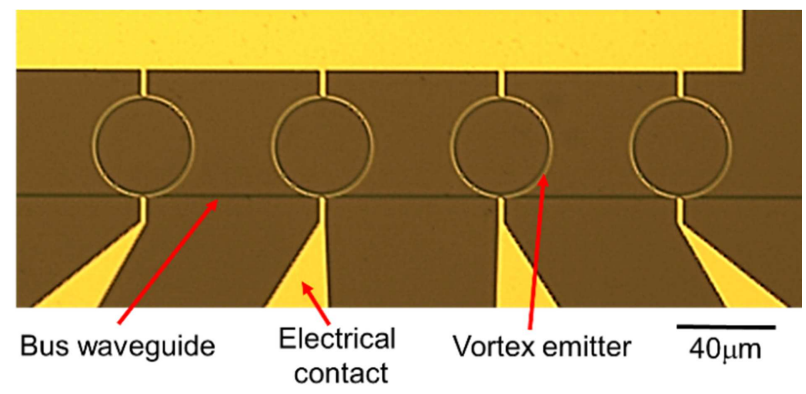

Fig. 1: Optical micrograph of an array of tunable optical vortex beam emitters.

The optical vortex beam emitter is based on a ring resonator with a grating perturbation on the inner radius of the ring [4]. The phase matching condition of the second order grating condition couples light propagating in plane into a vertically emitted mode carrying optical angular momentum. The OAM vector mode order is defined by the in-plane grating characteristics, the injected light wavelength and direction, and the modal index of the waveguide. The OAM mode order, $l$, can be given as $l=\frac{n_{e f f} L}{\lambda_{R}}-q$, where $n_{e f f}$ is the effective waveguide refractive index, $L$ is the cavity length, $\lambda_{R}$, is the resonant wavelength of the ring and $q$ is the number of scattering elements associated with the grating. The dissipation of electrical power in the heater element causes a local refractive index change in the waveguide and hence tunes the OAM mode order spectrum across the injection wavelength as shown in Fig. 2. At a constant injection wavelength, the detuning of the ring resonances due to electrical tuning causes the vortex beam emission spectrum to shift to longer wavelengths and up to five OAM mode orders are accessible within a $100 \mathrm{~mW}$ tuning range of the resistive heater element. Furthermore, active switching between these addressable modes was 
demonstrated with speeds up to $10 \mathrm{kHz}$, orders of magnitude faster than is currently available using bulk optical technologies.
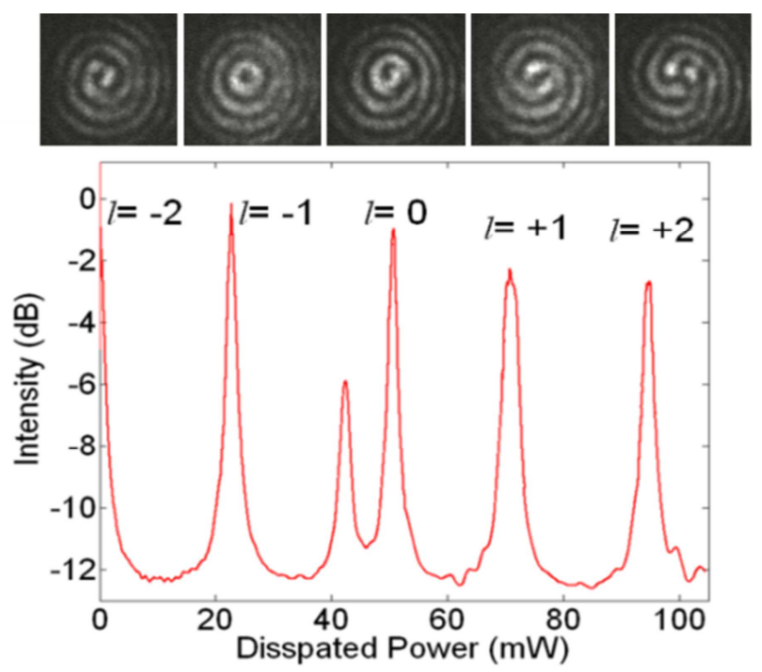

Fig. 2: Measured signal from a vortex beam emitter at $1556.35 \mathrm{~nm}$ with tuning of the waveguide refractive index through heater power dissipation. Above each resonance the measured interference pattern with a left-hand circularly polarised Gaussian beam is given.

Futhermore, if these optical vortex emitters are excited with replicas of the same optical signal, using bus waveguides placed at diametrically opposite points on the ring, then a vortex beam consisting of OAM mode orders of $\pm l$ can be excited simultaneously. By using the device geometry shown in Fig.3(a) the relative phase of these two signals can be varied, allowing rotation of the vortex beam interference pattern that is a function of the phase shift between the two arms of the interferometer, with potential applications in optical tweezing, cell sorting and manipulation[5]. Fig.3(b). shows simulations of the interference patterns generated by the beating of $\pm l$ mode orders with control excersied on the relative phase between the injected signals.

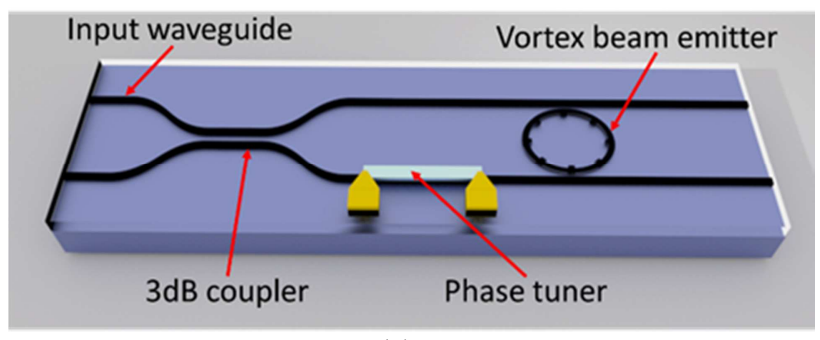

(a)

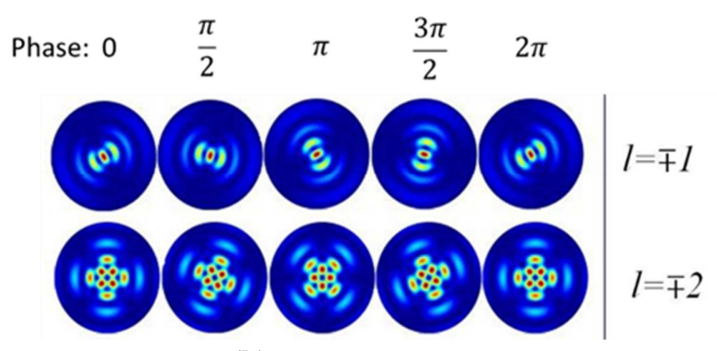

(b)

Fig. 3: (a) Schematic of an interference vortex beam emitter with relative phase control (b) emission interference patterns as a function of OAM mode order and relative phase

In conclusion, we demonstrate extremely compact optical vortex beam emitters with control over both the OAM mode order and the emitted beam intensity pattern using simple resistive heating elements as phase shifting components.

\section{References:}

[1] J. Wang, J. Yang, I. M. Fazal, N. Ahmed, Y. Yan, H. Huang, Y. Ren, Y. Yue, S. Dolinar, M. Tur, and A. E. Willner, "Terabit free-space data transmission employing orbital angular momentum multiplexing," Nat. Photonics, vol. 6, no. June, 2012

[2] M. Padgett and R. Bowman, "Tweezers with a twist," Nat. Photonics, vol. 5, no. 6, pp. 343-348, Jun. 2011.

[3] N. K. Fontaine, C. R. Doerr, and L. Buhl, "Efficient Multiplexing and Demultiplexing of Free-space Orbital Angular Momentum using Photonic Integrated Circuits," Opt. Fiber Commun. Conf., vol. 1, no. c, p. OTu1I.2, 2012.

[4] X. Cai, J. Wang, M. J. Strain, B. Johnson-Morris, J. Zhu, M. Sorel, J. L. O’Brien, M. G. Thompson, and S. Yu, "Integrated compact optical vortex beam emitters.," Science, vol. 338, no. 6105, pp. 363-6, Oct. 2012.

[5] J. E. Curtis and D. G. Grier, "Modulated optical vortices.," Opt. Lett., vol. 28, no. 11, pp. 872-4, Jun. 2003. 